\title{
Numerical Study on Internal Ballistics Characteristics of a Solid Propellant Rocket Motor
}

\author{
Saulius RAČKAUSKAS*, Algimantas FEDARAVIČIUS**, Arvydas SURVILA*** \\ * Kaunas University of Technology, Faculty of Mechanical engineering and Design, 54 Studentu Str., LT-51424 Kaunas, \\ Lithuania,E-mail: saulius.rackauskas@ktu.edu \\ **Kaunas University of Technology, Faculty of Mechanical engineering and Design, 54 Studentu Str., LT-51424 Kaunas, \\ Lithuania, E-mail: algimantas.fedaravicius@ktu.lt \\ ***Kaunas University of Technology, Faculty of Mechanical engineering and Design, 54 Studentu Str., LT-51424 Kaunas, \\ Lithuania, E-mail: arvydas.survila@ktu.lt \\ crossref http://dx.doi.org/10.5755/j01.mech.25.3.23742
}

\section{Introduction}

1.1. Significance of testing rocket propulsion devices using numerical models

Experimental testing, however attractive, is usually too expensive to be practical for research purposes in fields employing high value experimental samples. Therefore, computer models can be used as a substitute to achieve the same, if not more accurate results. Currently, the most common approaches are largely based on static firing tests [1]. The resulting empirical results allow for the evaluation and understanding of models and hypotheses in greater detail. However, the main drawbacks of static firing tests (high cost and labour intensity) limit the practicality of investigations into rocket propulsion technologies outside of government funded research [2]. However, the vast array of readily available computing power can be used to mimic static tests by running computer simulations. These simulations allow for the collection and processing of a greater array of data points, because everything is simulated in a mathematical plane and can be traced back or re-played on demand. Unfortunately, internal ballistics modelling is quite challenging and requires significant computational resources. It is still quite expensive, however with the ever-evolving cloud computing technology, numerical simulations are becoming an increasingly more attractive option over static tests.

As an example, one of the key differences of solid propellant motors is the ever-changing surface area of the combustion chamber walls, also called the evolution, erosion, or moving wall [3]. Meanwhile, the solid propellant burning surface changes throughout the combustion process with regards to its initial condition, which is based on the solid propellant grain geometry [4]. This process is presented in Fig. 1.

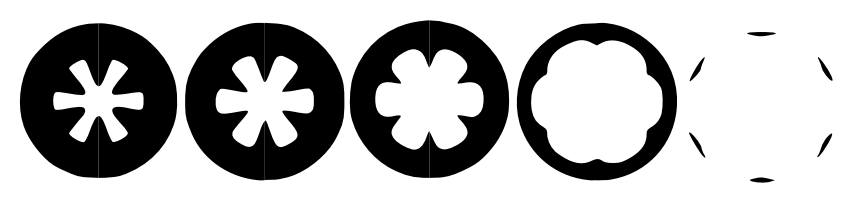

Fig. 1 Snapshot of the combustion of the star shaped solid propellant grain

It is noteworthy that this paper does not contain an investigation of the nozzle throat erosion, aging of the propellant [5] and only addresses the combustion process and its influence on the system. Owing to the reasons described above, the numerical methods used for computer modelling of rocket propulsion devices are different depending on the type of propellant.

\subsection{Increasing popularity of the practical use of numerical models}

Numerical models for rocket propulsion applications are becoming increasingly more affordable for small scale research and the private space industry. Increasingly more research is being performed by employing numerical methods for rocketry and space applications [6]. These developments have become available because of relatively cheap and abundant computational resources, which can be harnessed from computer clusters/clouds [7], local machines, or even low power computing clusters of various architectures [8]. For example, most of the research and development $(\mathrm{R} \& \mathrm{D})$ prototypes at the private space exploration company "SpaceX" were developed using numerical methods in the "cloud" rather than by static testing. This approach allows the saving of an enormous amount of labour and money, and in turn enables companies and researchers to expedite the iteration of propulsion system designs [9].

\subsection{Key challenges of numerical method-based simulations}

Despite the advantages of numerical method-based simulations, there are still significant challenges that must be 120 considered before employing this technique. The main issue of classical analytical methods is the need for homogeneous (linear) models, which is not a realistic assumption in fluid dynamics, and therefore does not represent the physics accurately. For this reason, numerical methodbased computer simulations are an attractive option, as they are capable of dealing with the chaotic nature of multiple, high energy reactions occurring simultaneously. However, numerical methods have inherent challenges that do not need to be considered in experimental approaches. One of the key challenges is the initialization of the solution, which requires the setting of all environmental parameters semisubjectively. This is important because the parameters must represent the environment in which the model has to operate in, and therefore a slight divergence from reality may render the simulation results useless and impossible to reproduce in the field. Another key challenge is the determination of the numerical schemes. Unfortunately, there is no "right" 
answer when it comes to choosing one, and thus, the simulation results can vary, on a case by case basis.

\section{Problem description}

\subsection{Motor description}

A rocket motor with a nominal average thrust of $12000 \mathrm{~N}$ (RM-12K) that uses solid propellants was used in this study. Furthermore, the RM- $12 \mathrm{~K}$ is also used in the rocket target with body diameter of $400 \mathrm{~mm}$ (RT-400) aerial target system [10] that was developed for the STINGER and GROM anti-aircraft missile defence systems [11]. The motor consists of seven main parts: 1. Igniter, 2. Pressure build up membrane, 3. Nozzle, 4. Solid propellant grain, 5. Case, 6 . Sealing rings and couplers, 7. Motor cap. Full composition of the rocket motor as depicted in Fig. 2 below.

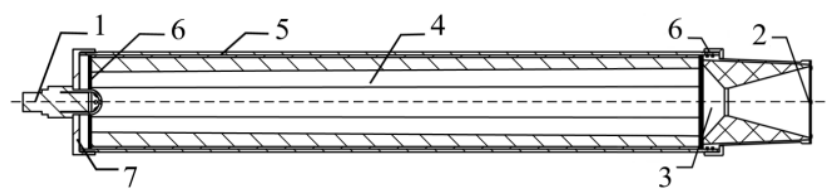

Fig. 2 3D CAD representation of the RM-12K

\subsection{Motor properties and composition}

The properties and composition of the motor had to be tailored and fine-tuned to achieve the desired motor characteristics. The RM-12K uses a solid rocket propellant with a specific grain geometry, which controls its combustion properties. The motor grain geometry was chosen as a neutral star type with an ammonium nitrate composite-based propellant (ANCP) forming the grain. Moreover, the propellant was designed specifically for this motor, using six main components. Those components were divided into 3 groups. 1 - oxidizer, 2 - fuel, 3 - binder. The chemical composition and other physical properties of the propellant are shown in Table 1.

Table 1

Molecular composition and physical properties of the propellant components

\begin{tabular}{|c|c|c|c|c|}
\hline Material & $\begin{array}{c}\text { Weight, } \\
\mathrm{g}\end{array}$ & $\mathrm{D}-\mathrm{H}$ & $\begin{array}{c}\text { Density, } \\
\mathrm{g} / \mathrm{cm}^{3}\end{array}$ & Composition \\
\hline $\begin{array}{c}\text { Ammo- } \\
\text { nium Ni- } \\
\text { trate }\end{array}$ & 55 & -1090 & 0.06230 & $4 \mathrm{H}-2 \mathrm{~N}-3 \mathrm{O}$ \\
\hline $\begin{array}{c}\text { Potassium } \\
\text { Perchlorate }\end{array}$ & 8 & -742 & 0.09100 & $\mathrm{~K}-\mathrm{Cl}-\mathrm{O} 4$ \\
\hline Aluminium & 14 & 0 & 0.09760 & $1 \mathrm{Al}$ \\
\hline Carbon & 2 & 0 & 0.06370 & $1 \mathrm{C}$ \\
\hline Silicone & 20 & -1820 & 0.03610 & $6 \mathrm{H}-2 \mathrm{C}-1 \mathrm{O}-1 \mathrm{Si}$ \\
\hline Iron Oxide & 1 & -1230 & 0.184 & $3 \mathrm{O}-2 \mathrm{Fe}$ \\
\hline
\end{tabular}

The density of propellant was $1623.4 \mathrm{~kg} / \mathrm{m}^{3}$. The simulation was performed using $100 \mathrm{~g}$ of propellant. The composite propellant consists of a number of gram atoms of each element presented in the ingredients (in ascending order): $0.012523 \mathrm{Fe}, 0.057738 \mathrm{Cl}, 0.057738 \mathrm{~K}, 0.269687 \mathrm{Si}$, $0.518903 \mathrm{Al}, 0.705888 \mathrm{C}, 1.374175 \mathrm{~N}, 2.580687 \mathrm{O}$, $4.366474 \mathrm{H}$.
From the simulation, the molecular weight of mixture was $23.896 \mathrm{~mol} / \mathrm{g}$ and gas specific heat was $2102 \mathrm{~J} / \mathrm{kg} / \mathrm{K}$. The performance calculations showed that the specific impulse of the designed ANCP propellant was $235 \mathrm{~s}$.

The Propellant Evaluation Program (PEP) software was used to obtain the chemical kinetics data. The software itself was developed for designing and analysing the composition and performance of propellants [12]. To avoid numerical complexities, the igniter and ignition period were excluded here. The ignition process was simulated with constant conditions for the temperature and density of the jet gas escaping from five jets, which mimicked igniter jet holes. The design process of the RM-12K involved custom tailoring of the properties and composition of the propellant grain and its shape. The motor model was designed using CAD software and exported as stereographic (STL) format models for future pre-processing.

\section{Model and methods}

\subsection{Mass conservation}

To solve the partial differential equations using numerical methods, assumptions must be made, and boundaries are introduced. The main equations are based on fluid dynamics and thermodynamic laws regarding physical continuities. Hence, conservation of mass (mass continuity equation), Eq. (1), describes how mass is conserved in the closed system [13].

$$
\begin{aligned}
& V_{f} \frac{d \rho}{d t}+\frac{d}{d x}\left(\rho v A_{y}\right)+R \frac{d}{d y}\left(\rho \omega A_{z}\right)+\xi \frac{\rho u A_{x}}{x}= \\
& =R_{D I F}+R_{S O R},
\end{aligned}
$$

where: $V_{F}$ is the fractional volume open to flow; $\rho$ is the fluid density; $R_{D I F}$ is the turbulent diffusion term; $R_{S O R}$ is the mass source term, and $\xi$-geometry reference frame type indicator. The velocity components $(u, v, w)$ are in the coordinate directions $\left(r, R_{S O R}, z\right) . A_{x}, A_{y}$, and $A_{z}$ are the area fractions for flow in the directions corresponding to the grid axis. In the cylindrical coordinate system, the y derivatives must be transformed into azimuthal derivatives. The conversion equation is given as follows:

$$
\frac{d}{d y} \rightarrow \frac{1}{r} \frac{d}{d \theta}
$$

\subsection{Fluid motion}

The equations of motion for the fluid velocity components $(\mathrm{u}, \mathrm{v}, \mathrm{w})$ in the three coordinate directions are given by the Navier-Stokes equations with additional terms capable of handling the specific problem represented in Eq. (3). Hence, the velocity magnitude can be derived by the relation $U=\sqrt{u^{2}+v^{2}+w^{2}}$. In this study, the velocity is measured in SI units [14]. 


$$
\begin{aligned}
& \frac{d u}{d t}+\frac{1}{V_{F}}\left\{u A_{x} \frac{d u}{d x}+v A_{y} R \frac{d u}{d y}+w A \frac{d u}{d z}\right\}-\xi \frac{A_{y} v^{2}}{x V_{F}}= \\
& =-\frac{1}{\rho} \frac{d p}{d x}+G_{x}+f_{x}-b_{x}-\frac{R_{S O R}}{\rho V_{F}}\left(u-u_{w}-\delta u_{s}\right) \frac{d v}{d t}+ \\
& +\frac{1}{V_{F}}\left\{u A_{x} \frac{d v}{d x}+v A_{y} R \frac{d v}{d y}+w A_{z} \frac{d v}{d z}\right\}+\xi \frac{A_{y} u v}{x V_{F}}= \\
& =-\frac{1}{\rho}\left(R \frac{d p}{d y}\right)+G_{y}+f_{y}-b_{y}-\frac{R_{S O R}}{\rho V_{F}}\left(v-v_{w}-\delta v_{s}\right) \times \\
& \times \frac{d w}{d t}+\frac{1}{V_{F}}\left\{u A_{x} \frac{d w}{d x}+v A_{y} R \frac{d w}{d y}+w A_{z} \frac{d w}{d z}\right\}= \\
& =-\frac{1}{\rho} \frac{d p}{d z}+G_{z}+f_{z}-b_{z}-\frac{R_{S O R}}{\rho V_{F}}\left(w-w_{w}-\delta w_{s}\right)
\end{aligned}
$$

here: $\left(G_{x}, G_{y}, G_{z}\right)$ are the body accelerations; $\left(f_{x}, f_{y}, f_{z}\right)$ are the viscous accelerations; $\left(b_{x}, b_{y}, b_{z}\right)$ are the introduced masses at the source represented by geometric components; $\left(u_{w}, v_{w}, w_{w}\right)$ represents the velocity of the source component, and $\left(u_{s}, v_{s}, w_{s}\right)$ is the velocity component of the fluid at the surface of the source relative to the source itself. The derivation of this source term is given by:

$$
U_{s}=\frac{d Q}{\rho Q d A} n,
$$

where: $d Q$ is the mass flow rate; $\rho_{Q}$ is the fluid source density; $d A$ is the area of the source surface in the cell, and $n$ is the outward normal to the surface. In this case $n=0.0$, because the flow is a stagnant pressure type. This means that the source term, which introduces fluid into the domain, has a zero-velocity magnitude at the interface of the propellant surface. As a result, pressure must build up at the source to move the fluid away from the source. This helps to model the emerging fluid from the propellant grain and mimic the grain burning process when emerging fluid creates momentum inside the source component.

\subsection{Heat transfer}

The fluid energy equations Eq. (5) are required to calculate the heat transfer of the fluid. The fluid temperature affects the fluid flow properties during the transition from the combustion chamber, through the throat of the nozzle, before escaping the choked flow into the diverging part of the nozzle, when leaving the domain.

$$
\begin{aligned}
& V_{F} \frac{d}{d t}(\rho I)+\frac{d}{d x}\left(\rho I u A_{x}\right)+R \frac{d}{d y}\left(\rho I v A_{y}\right)+\xi \frac{\rho I u A_{x}}{x}= \\
& =-p\left\{\frac{d u A_{x}}{d x}+R \frac{d v A_{y}}{d y}+\frac{d w A_{z}}{d z}+\xi \frac{u A_{x}}{d x}\right\}+R I_{D I F}+ \\
& +T_{D I F}+R I_{S O R},
\end{aligned}
$$

here: $I$ is the macroscopic mixture internal energy where the subscripts DIF and SOR refers to the diffusion and source terms, respectively. Because the model employs a two-fluid problem, $\rho I$ is given by:

$$
\rho I=F \rho_{1} I_{1}+(1-F) \rho_{2} I_{2}
$$

The heat conduction diffusion process is introduced in Eq. (7).

$$
\begin{aligned}
& T_{D I F}=\frac{d}{d x}\left(k A_{x} \frac{d T}{d x}\right)+R \frac{d}{d y}\left(k A_{y} R \frac{d T}{d y}\right)+ \\
& +\frac{d}{d z}\left(k A \frac{d T}{d z}\right)+\xi \frac{k A_{x} T}{x}
\end{aligned}
$$

here: $k$ is the thermal conductivity. For this model each fluid is locally weighted by the fluid volume fraction $F$.

\subsection{Fluid interface}

To model the burning surface of the solid propellant, the fluid interface in the computational cell between the fluid and solid domains must be introduced. Such phenomena can be defined in terms of the volume of fluid function, $F(x, y, z, t)$ [15]. This function represents the volume of fluid per unit volume and is given by Eq. (8). This interface moves during the simulation, and its parameters are derived by Eq. (9).

$\frac{d F}{d t}+\frac{1}{V_{F}}\left[\begin{array}{l}\frac{d}{d x}\left(F A_{x} u\right)+R \frac{d}{d y}\left(F A_{y} v\right)+ \\ +\frac{d}{d z}\left(F A_{z} w\right)+\xi \frac{F A_{x} u}{x}\end{array}\right]=F_{D I F}+F_{S O R}$

where:

$$
F_{D I F}=\frac{1}{V_{F}}\left\{\begin{array}{l}
\frac{d}{d x}\left(v_{F} A_{x} \frac{d F}{d x}\right)+R \frac{d}{d y}\left(v_{F} A_{y} R \frac{d F}{d y}\right)+ \\
+\frac{d}{d z}\left(v_{F} A_{z} \frac{d F}{d z}\right)+\xi \frac{v_{F} A_{x} F}{x}
\end{array}\right\}
$$

here the diffusion coefficient is defined as $v_{F}=\frac{c_{F} \mu}{\rho}$, where $c_{F}$ is a constant whose reciprocal is sometimes referred to as the turbulent Schmidt number, and $F_{S O R}$ is the density source (corresponding to $R_{S O R}$ in Eq.(1)). For a compressible twofluid simulation, $F$ represents the volume fraction of fluid, and the density is calculated from the compressible fluid equation-of-state.

\subsection{Numerical schemes}

Second-order schemes were used for the approximations of the model equations. First order schemes were used only to check the model's integrity and stability in the first stages. The final simulations were conducted using only second-order, monotonicity- preserving upwind difference method-based schemes. These schemes require greater computational resources, however, are substantially more precise, compared with the first-order schemes. The monotonicity-preserving method was applied to approximate momentum, density, energy, and fluid fraction advection. The discretization scheme is derived using second order polynomial approximations to the advected quantity in each of the coordinate directions [16]. Eq. (10) describes the advection 
of variable $Q$ in the x-direction, providing the fluxed value through a cell-face $Q^{*}$.

$$
Q^{*}=Q_{i}+\frac{1}{2} A(1-C) \delta x_{i}
$$

The coefficient $A$ can be calculated from two neighbouring first derivatives by linear interpolation, provided these derivatives are second-order accurate. The latter can be achieved by computing the derivatives at the midpoints between the $Q_{i}$ locations, such as that described in Eq. (11).

$$
\frac{d Q}{d x_{i+\frac{1}{2}}}=2 \frac{Q_{i+1}-Q_{i}}{\delta x_{i+1}+\delta x_{i}}
$$

Eq. (11) is a second-order accurate first derivative for $Q$ at the point between $Q_{i}$ and $Q_{i}+1$. To ensure monotonicity, it is necessary to restrict the value of the derivative A [17]. The value of $A$ is not allowed to exceed twice the minimum magnitude of the centred $Q$ derivatives used in its computation:

$$
A \leq 2\left(\frac{d Q}{d x_{i}}, \frac{d Q}{d x_{i+1}}\right) .
$$

The momentum advection second-order approximation allows for stable convergence even for unstable, swirling flow. The same second-order schemes for the density and heat transfer equations, avoid the issues of the firstorder schemes, such as numerical diffusion.

\subsection{Combustion}

The simulation was executed without coupling of the direct chemical kinetics modelling. All of the required values for the material properties and coefficients were obtained from a separate investigation, briefly described in the first part of this paper 2. The combustion rate and energy production equations are given by Eqs. $(13,14)$, respectively [18].

$$
Q_{M}=\rho_{\text {solid }}\left(a P^{b}\right)
$$

here: $Q_{M}$ is the combustion gas mass flow rate in $\mathrm{kg} / \mathrm{s}$ at the gas/propellant boundary; $\rho_{\text {solid }}$ is the density of the propellant in $\mathrm{kg} / \mathrm{m}^{3} ; P$ is the pressure of the gas at the solid/gas interface, a is the burning coefficient (empirical parameter), and $\mathrm{b}$ is the burning exponent (empirical parameter).

$$
Q_{E}=Q_{M} C_{P} T_{b u r n}
$$

here: $Q_{E}$ is the energy produced by the reaction in Joules; $Q_{M}$ is the combustion flow rate in $\mathrm{m} / \mathrm{s} ; C_{P}$ is the specific heat of the gas at constant pressure, and $T_{\text {burn }}$ is the propellant burning temperature obtainendedm the chemical kinetic calculations.

duced:
Using this model some limitations must be intro-
1. No additional source terms are introduced into the momentum equations because the combustion is assumed to be of the stagnation type.

2. The combustion gas density is calculated from the ideal gas equation.

3. Direct turbulence effects on the burn rate are ignored.

4. The stress and deformation calculations for the propellant grain are ignored.

The model combustion component is described by a stationary component but changes shape and volume according to the evolution of time. These changes are constrained by the initial conditions of the combustion component properties and initial shape of the propellant grain. The combustion component area and volume fractions are computed at the single cell level, for every time step during the simulations until the combustion component is depleted in the computational domain. The primary variable that represents the geometry component is the relative (fractional) volume of the solid in a cell, which is the ratio of the solid volume in the cell to the total cell volume. This relationship is described by Eq. (15) [19].

$$
V_{f, \text { combust }}=\frac{V_{\text {combust }}}{V_{\text {cell }}}, 0 \leq V_{f, \text { combust }} \leq 1
$$

The open volume fraction in a cell, or volume fraction, can then be described in the form of Eq. (16).

$$
V_{f}=1-V_{f, \text { combust }} \text {. }
$$

The equation for the change in the solid propellant content in the computational cell can be written in the form of Eq. (17), where the open area of the burning surface of the solid propellant of the cell is $d A$.

$$
\frac{d V_{f, \text { combust }}}{d t}=\frac{Q_{M} d A}{\rho_{\text {solid }} V_{\text {cell }}} .
$$

Fig. 3 illustrates the interface region used in computation for the combustible element, where the control volume is the computational domain mesh element (cell), and fractional parts of the solid propellant $\left(V_{\text {solid }}\right)$ and fluid domain fraction (gas).

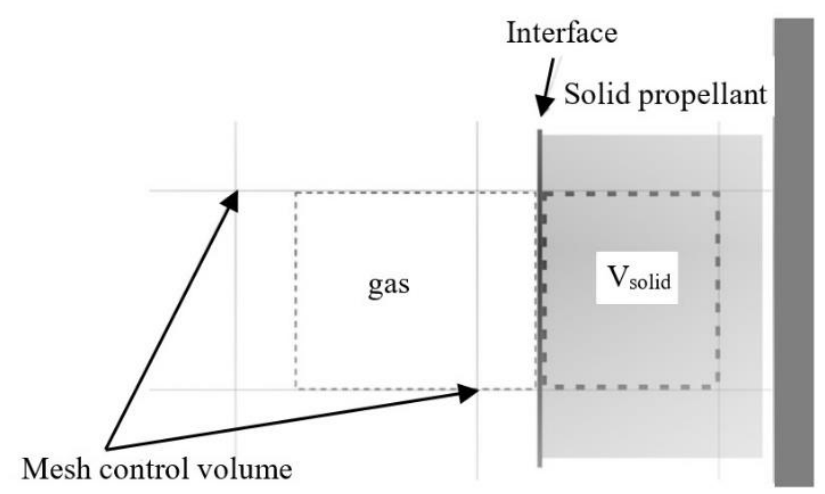

Fig. 3 Schematic of the interface region in the numerical

This model provides the amount of propellant burned in each time step, described by Eq. (18). 


$$
\frac{d M}{d t}=Q_{M} \frac{d A}{d t}
$$

The energy produced by the combustion process is distributed over the gas volume at the burning surface interface. After introducing the processes described above, the diffusion and convection processes are calculated, and the mass transport equation is solved for the gas produced by the reaction. This equation can be described by the mass source relation for the combustion chamber pressure:

$$
\frac{d m}{d t}=a P^{b}
$$

\section{Model setup}

\subsection{Model key assumptions}

The following assumptions and constraints are used for the numerical method-based computer simulation. The simulation was executed using an 8-core workstation grade machine. The simulation type was set to transient, and the physical end time was set to $5 \mathrm{~s}$. This was performed with an additional adaptive time step term. This approach allowed the capturing of the entire combustion process, until the propellant was completely depleted. Moreover, a coarsen time step was chosen to expedite the simulation because of the time required to calculate the changing computational domain interface, which limits the simulation progress. Moreover, the initial time step was set to $245 \mathrm{~ns}$. This value was chosen from the results of the previous simulation, which was the most time saving time step to provide stable convergence, and the time savings of the initial phase of the simulation. Furthermore, the minimum and maximum time step sizes were set to $1 \mathrm{~ns}$ and $0.1 \mathrm{~s}$, respectively. The maximum time step was chosen to capture the final moments of the simulation when the combustion process was already over. The total computation time for one simulation, comprising 170 million iterations, was approximately $2571000 \mathrm{~s}$. In conclusion, the assumptions and constraints of the simulation were designed to optimize the time taken to complete the simulation, while maintaining an acceptable result accuracy. As a result, the simulation required $715 \mathrm{~h}$ of computing time on average using an 8-core machine.

\subsection{Geometry and mesh setup}

The imported geometry and computational mesh setup had the following properties. The model was cylindrical and axisymmetric; meshed with structured hexahedral cells in a cylindrical reference frame. Moreover, the computational domain height and dimeter were 1.12 and $0.2 \mathrm{~m}$, respectively, with a computational cell count of 162000 . For post processing purposes, two cutting planes were used to represent the simulation results. The first cut was made across the centre axis of the motor, providing a representation and visualization of the combustion process inside the rocket motor. A second cut was made perpendicular to the first cut to extract the cross-sectional view of the rocket motor. Both cutting plane locations are illustrated in fig. 4. Furthermore, two probes were created and strategically positioned to allow the simulation to capture and record specific data values. One probe point was constrained to the same position as the pressure probe in the real-life experiment that was completed prior to the simulation. This probe was labelled Probe 1 and placed near the head section. A second probe was placed at the very end of the nozzle and was labelled Probe 2 . The setup was designed to effectively capture and monitor the simulation data, for the purpose of comparison with the results obtained by previously performed static tests.

\subsection{Initial simulation conditions}

The initial conditions for the simulation were constrained to the fluid domain with a $300 \mathrm{~K}$ initial air temperature inside the combustion chamber and 1.01325 bar (101325 Pa) uniform atmospheric pressure. Moreover, a mass momentum source element was created to represent the propellant igniter. This artificial source had to mimic the real ignition process, and therefore, the same combustion gas properties were used as those of an actual propellant. Furthermore, the temperature, flow rate, and gas density were $1200 \mathrm{~K}, 0.02 \mathrm{~kg} / \mathrm{s}$, and $10 \mathrm{~kg} / \mathrm{m}^{3}$, respectively. The igniter/mass source surface dimeter was $0.01 \mathrm{~m}$. There were five surfaces in total. One of the mass sources was placed perpendicular to the domain axis, and the other four were positioned in a cylindrical pattern around the domain axis, equally spaced at $45^{\circ}$ angles. Finally, the igniter was placed near the Probe 1 location with a 0.4 s ignition time. The initial conditions were chosen to closely represent the experimental environment.

\subsection{Boundary conditions}

The boundary conditions are very important for obtaining the desired simulation results. The boundary conditions for the cylindrical domain were constrained with the same symmetry for all boundaries, except for the periodic boundaries in contact with each other, which is necessary in cylindrical meshes when the full domain is simulated. Furthermore, to achieve a stable convergence, the boundary at the end of the nozzle was constrained as a pressure outlet. To conclude, this relatively simple set up of boundary conditions is all that is required to obtain the physical results.

\subsection{Setup for a specific numerical code}

Some key assumptions had to be considered for the specific numerical code. The discussion below describes these assumptions and the reasoning behind them. Two computational domains, solid and fluid, were constrained with different additional properties for different geometric parts. All of the properties for the fluid and solid domains required for the simulation are described in Table 3.

Which could possibly develop issues regarding information transfer between the centres of the cells. Finally, all numerical schemes for the final simulations were explicitly executed. For time saving purposes, an adaptive time step was introduced into the simulation. Gravity was also introduced into the model. A graphic representation of the model in the Flow-3D graphical user interface is shown in Fig. 4. The arrow pointing towards the right indicates the gravity vector constraint. This indicates that static firing testing was executed having motor in the same position as described below. 
Table 3

Main properties of the fluid and solid domains, as well as those of the introduced combustion gas as a mass source for the simulation

\begin{tabular}{|c|c|c|c|c|}
\hline Fluid & $\begin{array}{c}\text { Density } \\
\rho, \mathrm{kg} / \mathrm{m}^{3}\end{array}$ & $\begin{array}{c}\text { Individual } \\
\text { gas const. } \\
R_{t}, \mathrm{~J} / \mathrm{kg} / \mathrm{K}\end{array}$ & $\begin{array}{c}\text { Specific } \\
\text { heat } C_{v}, \\
\mathrm{~J} / \mathrm{kg} / \mathrm{K}\end{array}$ & $\begin{array}{c}\text { Thermal } \\
\text { conduc- } \\
\text { tivity } k, \\
\mathrm{~W} / \mathrm{m} / \mathrm{K}\end{array}$ \\
\hline $\begin{array}{c}\text { Combus- } \\
\text { tion gas }\end{array}$ & 1.446 & 347.943 & 2102 & 0.1 \\
\hline Solid & $\begin{array}{c}\text { Density } \\
\rho, \mathrm{kg} / \mathrm{m}^{3}\end{array}$ & $\begin{array}{c}\text { Combus- } \\
\text { tion tem- } \\
\text { perature } \\
T_{c}, \mathrm{~K}\end{array}$ & $\begin{array}{c}\text { Combus- } \\
\text { tion } \\
\text { threshold } \\
\text { tempera- } \\
\text { ture } T_{t}, \mathrm{~K}\end{array}$ & $\begin{array}{c}\text { Empirical } \\
\text { coeffi- } \\
\text { cients } a, b\end{array}$ \\
\hline $\begin{array}{c}\text { Propel- } \\
\text { lant }\end{array}$ & 1623 & 2322 & 1000 & 0.039, \\
0.27
\end{tabular}

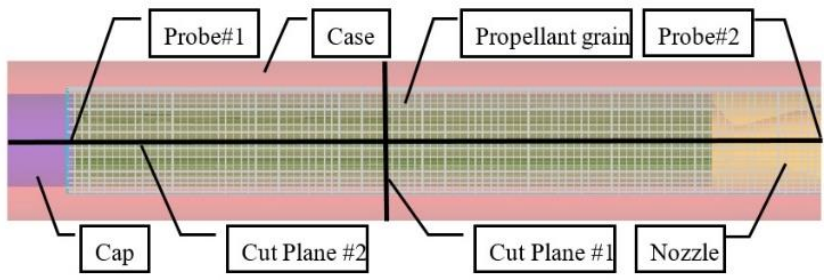

Fig. 4 Model schematic

In conclusion, it is noteworthy, that different assumptions may be required if our results are replicated using a different numerical code.

\section{Results}

\subsection{Method for capturing the simulation results}

The methodology for capturing and presenting the simulation results was determined as follows. First, for the graphical representation, the simulation results were captured for six different time marks: $1-$ initial state at $t=0 \mathrm{~s}$, $2-1 / 5$ of the finished combustion process at time $t=1 \mathrm{~s}, 3$ $-2 / 5$ of the initial combustion process at time $t=2 \mathrm{~s}, 4-3 / 5$ of the combustion process at time $t=3 \mathrm{~s}, 5-4 / 5$ of the combustion process at time $t=4 \mathrm{~s}$, and 6 - finished combustion process at time $t=5 \mathrm{~s}$. This was performed to clearly represent the changing evolution of a solid propellant grain throughout time. Second, the data used to plot the results were obtained from the probe points and mesh results. Moreover, the data for the burn distance and open area of the combusting grain surface and volume were obtained from the results of the mesh dependent history. Meanwhile, the pressure and temperature were extracted from the probes. Additional data were obtained, representing the thrust and total impulse of the motor. Finally, all of the data were captured from the very beginning of the simulation, only skipping the igniter execution time of $0.4 \mathrm{~s}$. Most importantly, the pressure and temperature data were simultaneously collected by all during the simulation.

\subsection{Discussion of the results}

After post processing the simulation data, the results were obtained. The probe sample points for the pressure and temperature are provided in Figs. 5, 6, 7, and 8.

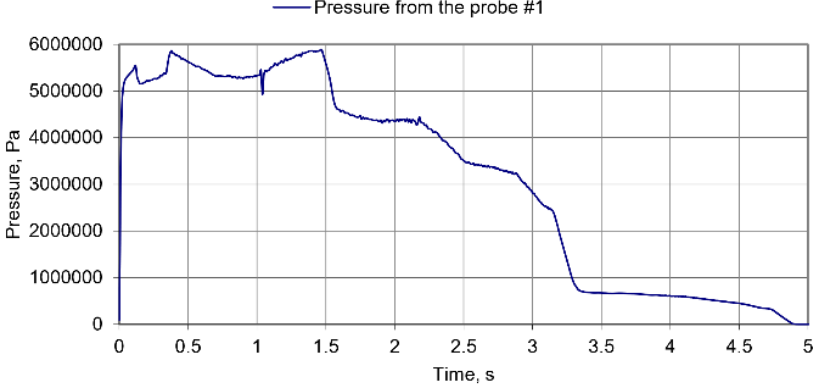

Fig. 5 Pressure versus time for the Probe 1 point (cap)

The pressure probing of the cap section was performed at the same location as for the experiment. The plotted results of the Probe 1 exhibited a standard pressure curve behaviour for a star shaped propellant grain type. The pressure readings for different time marks were: 1 - initial state at $t=0 \mathrm{~s}, 101325 \mathrm{~Pa}(1.01 \mathrm{bar}) ; 2-1 / 5$ of the finish combustion process at time $t=1 \mathrm{~s}, 5339105 \mathrm{~Pa}$ (53.39 bar); 3 $2 / 5$ of the initial combustion process at time $t=2 \mathrm{~s}, 4364912$ $\mathrm{Pa}$ (43.65 bar); $4-3 / 5$ of the combustion process at time $t=$ $3 \mathrm{~s}, 2757237 \mathrm{~Pa}$ (27.57 bar); $5-4 / 5$ of the combustion process at time $t=4 \mathrm{~s}, 605743 \mathrm{~Pa}(6.06 \mathrm{bar})$; and 6 - finished combustion process at time $t=5 \mathrm{~s}, 446 \mathrm{~Pa}(0.0045 \mathrm{bar})$.

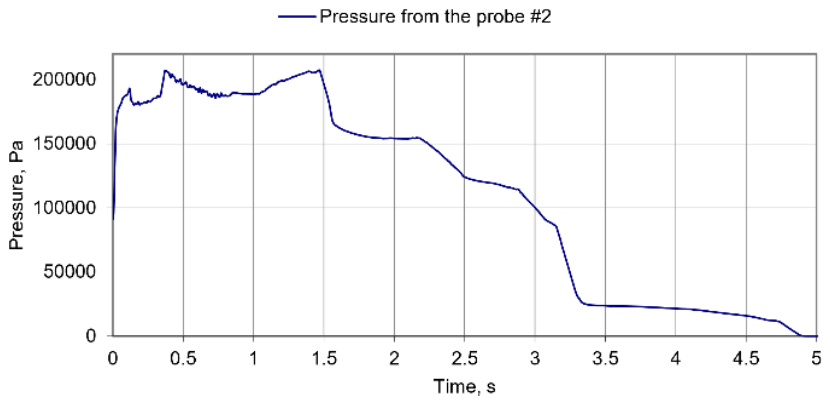

Fig. 6 Pressure versus time for the Probe 2 point (nozzle)

For the Probe 2 point, the pressure readings exhibited a similar pattern as that of the cap section, but with lower values, which was caused by the converging - diverging nozzle. In the experiment, pressure readings at the Probe 2 point were not possible because of the volatile nature of the combusting propellant, which limited the data measurement options during the static motor test. The pressure readings of the Probe 2 point were: 1 - initial state at $t=0 \mathrm{~s}$, $91192 \mathrm{~Pa}$ (0.91 bar); $2-1 / 5$ of the finish combustion process at time $t=1 \mathrm{~s}, 188887 \mathrm{~Pa}(1.89 \mathrm{bar}) ; 3-2 / 5$ of the initial combustion process at time $t=2 \mathrm{~s}, 154102 \mathrm{~Pa}(1.54$ bar); $4-3 / 5$ of the combustion process at time $t=3 \mathrm{~s}, 97795$ $\mathrm{Pa}$ (0.98 bar); $5-4 / 5$ of the combustion process at time $t=$ $4 \mathrm{~s}, 21330 \mathrm{~Pa}(0.21 \mathrm{bar})$; and 6 - finished combustion process at time $t=5 \mathrm{~s}, 14.31 \mathrm{~Pa}(0.00014 \mathrm{bar})$.

The temperature readings were only obtained from the simulations. The temperature probes used in the static test were unable to capture reliable data because the temperature was too high and exposure time too long of the hot combustion gas. At the Probe 1 location, the temperature readings were: 1 - initial state at $t=0 \mathrm{~s}, 300 \mathrm{~K} ; 2-1 / 5$ of the finish combustion process at time $t=1 \mathrm{~s}, 2359 \mathrm{~K} ; 3-2 / 5$ of the initial combustion process at time $t=2 \mathrm{~s}, 2360 \mathrm{~K} ; 4-$ $3 / 5$ of the combustion process at time $t=3 \mathrm{~s}, 2350 \mathrm{~K} ; 5-$ $4 / 5$ of the combustion process at time $t=4 \mathrm{~s}, 2338 \mathrm{~K}$, and 6 - finished combustion process at time $t=5 \mathrm{~s}, 1895 \mathrm{~K}$. 


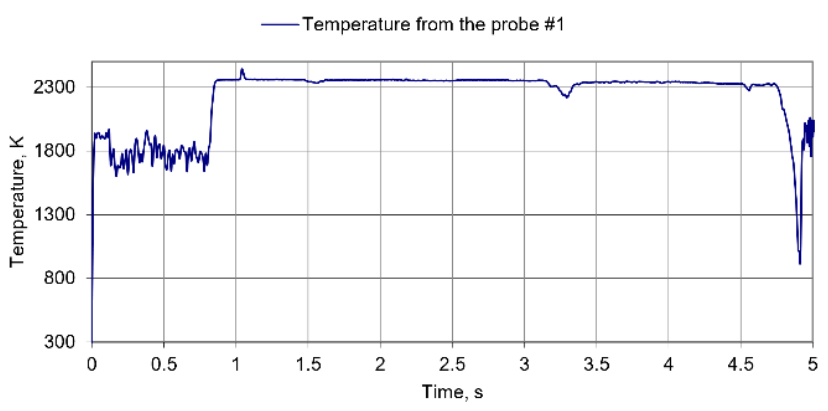

Fig. 7 Temperature versus time for the Probe 1 point (cap)

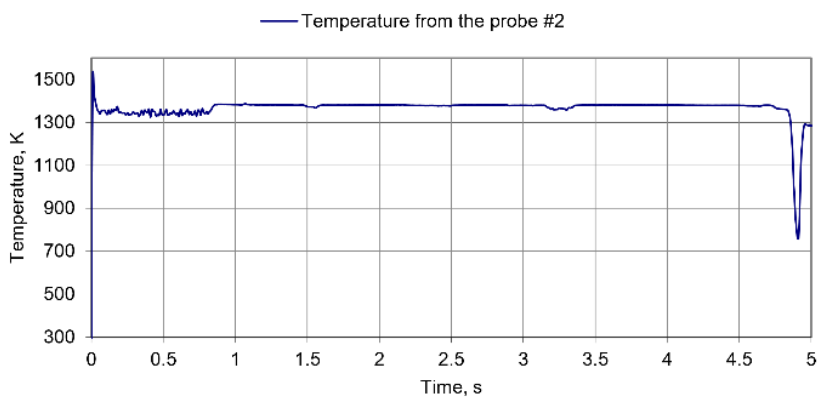

Fig. 8 Temperature versus time for Probe 2 point (nozzle)

The temperature sampling data of Probe 2 were different from that of Probe 1 . This was caused by the combustion gas escaping from the combustion chamber through the nozzle throat. As a result, heat energy was released because of the converging-diverging nozzle. The temperature data readings of Probe 2 were: 1 - initial state at $t=0 \mathrm{~s}, 300$ $\mathrm{K} ; 2-1 / 5$ of the combustion process at time $t=1 \mathrm{~s}, 1382 \mathrm{~K}$; $3-2 / 5$ of the initial combustion process at time $t=2 \mathrm{~s}, 1382$ $\mathrm{K} ; 4-3 / 5$ of the combustion process at time $t=3 \mathrm{~s}, 1380 \mathrm{~K}$; $5-4 / 5$ of the combustion process at time $t=4 \mathrm{~s}, 1381 \mathrm{~K}$; and 6 - finished combustion process at time $t=5 \mathrm{~s}, 1285 \mathrm{~K}$.

Additional data were extracted for the mesh dependent results to better represent and enhance the understanding of the problem. The average fluid temperatures in the computational domain, open surface area of the propellant grain, and volume of the propellant grain are plotted in Figs. 9, 10, and 11, respectively

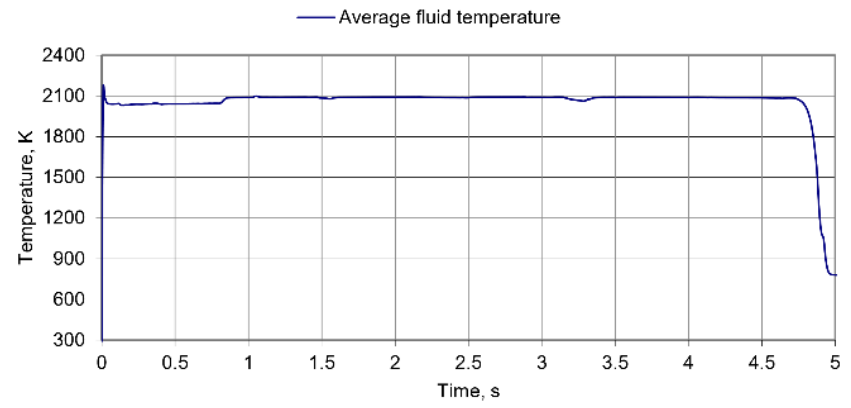

Fig. 9 Average fluid temperature

The sample data of the average fluid temperature were obtained from the whole computational domain. This was performed to determine the required thermal protection solutions. High temperatures inside the combustion chamber can lead to failure of the mechanical components due to high thermal loads. From the sampled data, the average fluid temperature inside the combustion chamber was $2080 \mathrm{~K}$, which demonstrates that adequate thermal protection is necessary to obtain nominal motor performance parameters and avoid catastrophic failures due to component rupturing.

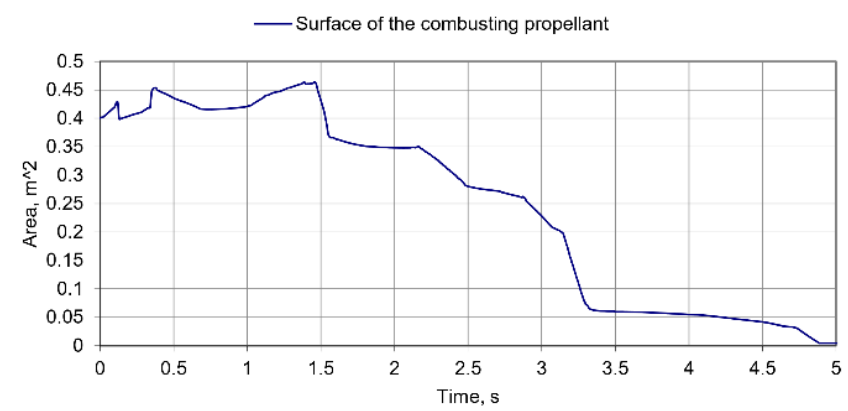

Fig. 10 Combustion surface area

The performance of the motor is deeply coupled with its combustion surface area. This is a key parameter when designing rocket motors for specific performance requirements. The open surface of the solid propellant rocket motor from the beginning of combustion until the propellant was depleted completely, is shown in the plot above (Fig 10). The values of the open surface of the propellant grain were: 1 - initial state at $t=0 \mathrm{~s}, 0.4 \mathrm{~m}^{2} ; 2-1 / 5$ of the combustion process at time $t=1 \mathrm{~s}, 0.42 \mathrm{~m}^{2} ; 3-2 / 5$ of the initial combustion process at time $t=2 \mathrm{~s}, 0.348 \mathrm{~m}^{2} ; 4-3 / 5$ of the combustion process at time $t=3 \mathrm{~s}, 0.224 \mathrm{~m}^{2} ; 5-4 / 5$ of the combustion process at time $t=4 \mathrm{~s}, 0.054 \mathrm{~m}^{2}$; and 6 - finished combustion process at time $t=5 \mathrm{~s}, 0.005 \mathrm{~m}^{2}$.

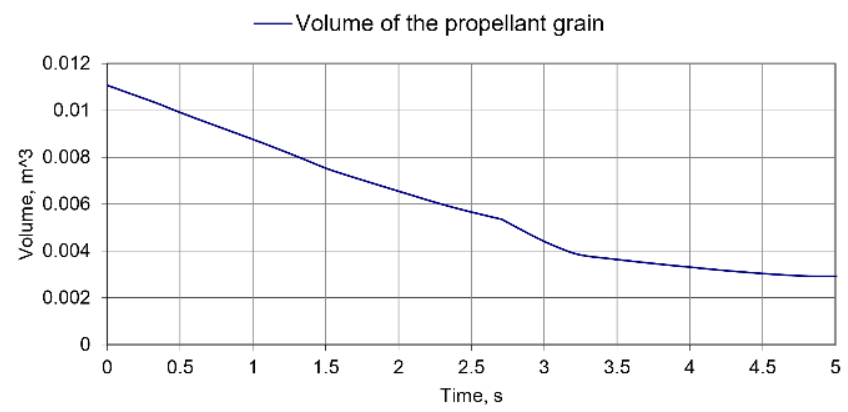

Fig. 11 Combustion surface volume

To obtain a greater understanding of the results, the change in the combustion propellant grain volume was plotted in Fig.11. The combustion stability was determined, which is based on a stable regression of the propellant volume during combustion. According to these results, the combustion was stable during the entire combustion until the sliver phase was reached, and then continued steadily until the propellant was depleted completely. The volume of the propellant steadily depleted from the initialization $\left(0.011 \mathrm{~m}^{3}\right)$, to the sliver phase $\left(0.0043 \mathrm{~m}^{3}\right)$.

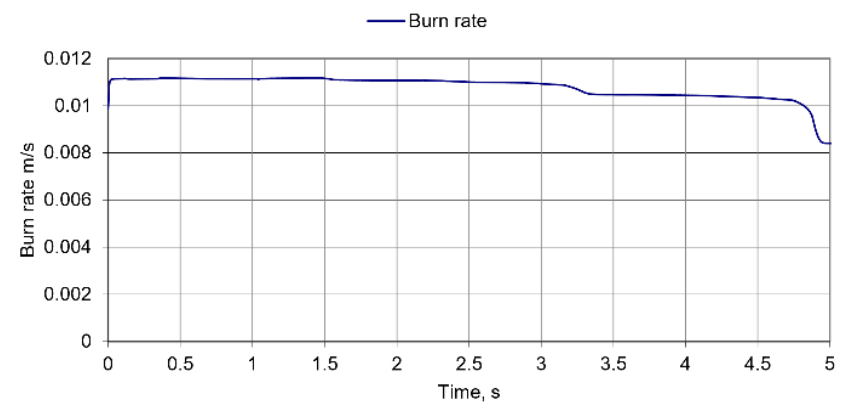

Fig. 12 Propellant grain burning rate

The burning rate of the propellant was extracted according to Eq. 20 as follows: $1-$ initial state at $t=0 \mathrm{~s}$, $0.00986 \mathrm{~m} / \mathrm{s} ; 2-1 / 5$ of the combustion process at time $t=1$ 
$\mathrm{s}, 0.0111 \mathrm{~m} / \mathrm{s} ; 3-2 / 5$ of the initial combustion process at time $t=2 \mathrm{~s}, 0.011 \mathrm{~m} / \mathrm{s} ; 4-3 / 5$ of the combustion process at time $t=3 \mathrm{~s}, 0.011 \mathrm{~m} / \mathrm{s} ; 5-4 / 5$ of the combustion process at time $t=4 \mathrm{~s}, 0.01 \mathrm{~m} / \mathrm{s}$; and 6 - finished combustion process at time $t=5 \mathrm{~s}, 0.0084 \mathrm{~m} / \mathrm{s}$.

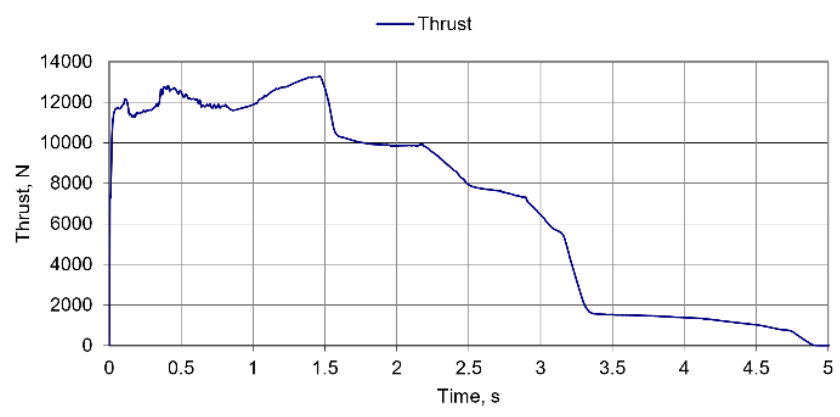

Fig. 13 Rocket motor thrust

The thrust produced by the motor is given by Eq. (20), and is explained below.

$$
F=\dot{m} v_{e}\left(p_{e}-p_{0}\right) A_{e}
$$

here: $F$ is the thrust; $\dot{m}$ is the mass flow rate from the nozzle exit; $v_{e}$ is the exit velocity from the nozzle (custom monitoring plane); $p_{e}$ is the pressure at the end of the nozzle (Probe $2) ; p_{0}$ is the atmospheric pressure (initial pressure), and $A_{e}$ is the exit area (custom monitoring plane). The thrust values were as follows: $1-$ initial state at $t=0 \mathrm{~s}, 0 \mathrm{~N} ; 2-1 / 5$ of the combustion process at time $t=1 \mathrm{~s}, 11879 \mathrm{~N} ; 3-2 / 5$ of the initial combustion process at time $t=2 \mathrm{~s}, 9859 \mathrm{~N} ; 4-3 / 5$ of the combustion process at time $t=3 \mathrm{~s}, 6359 \mathrm{~N} ; 5-4 / 5$ of the combustion process at time $t=4 \mathrm{~s}, 1364 \mathrm{~N}$; and 6 - finished combustion process at time $t=5 \mathrm{~s}, 1.02 \mathrm{~N}$.

Figs. 14 and 15 depict the evolution of the propellant grain during combustion at different times. The key advantages of being able to monitor and measure the changes in the propellant surface area and its shape over time is that the inner workings of the motor can be understood, and the parameters fine-tuned for a better performance. Moreover, the data provided by the cross section would not otherwise be available if only static testing had been performed. Furthermore, as shown in Fig. 14 the propellant grain transforms as it combusts. The vector field is included to represent the fluid motion. The data are also colour coded to represent the different components within the RM-12K motor.

\subsection{Model validation}

The model used for the simulation was valid and physically correct according to the observed parameters during the static testing and correlated within a reasonable degree of confidence to the parameters obtained during the simulation. The experimental results of the changes in pressure and thrust over time were evaluated (Fig. 16). Moreover, the aforementioned data were compared with the results obtained from the simulation. This was performed to test the model compatibility, and from the observed results, it can be concluded that the model is capable of representing all of the key characteristics of the combustion process of the solid propellant rocket motor.

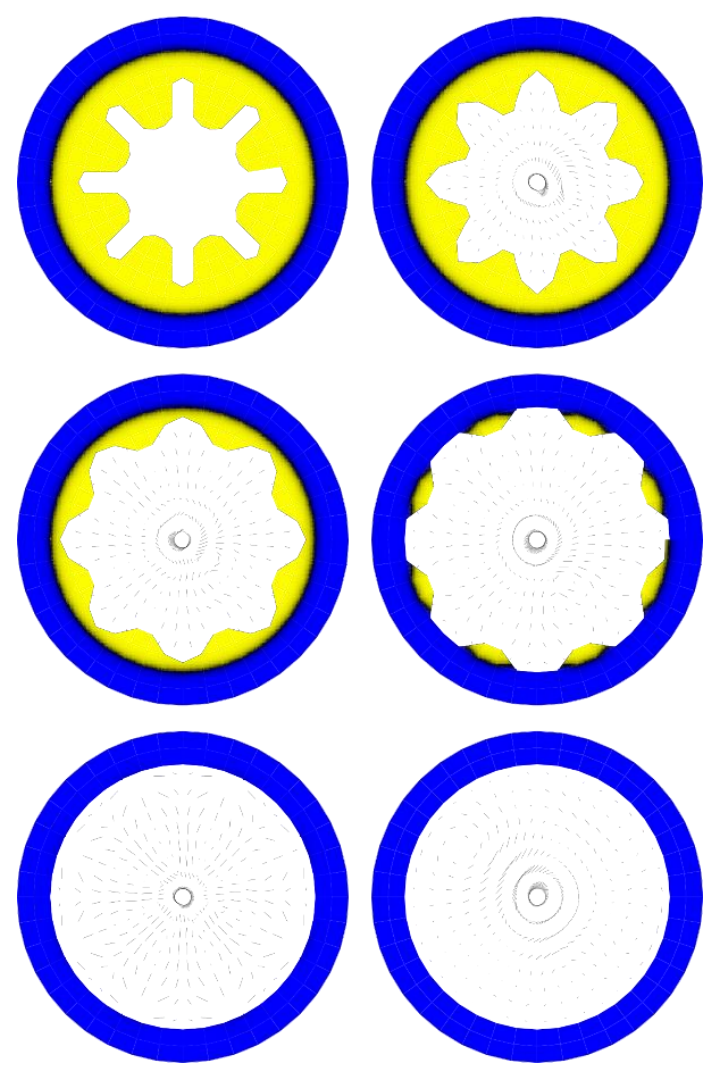

Fig. 14 Propellant combustion evolution at $t=0,1,2,3,4$, and 5 seconds for the $x-y$ axis

Fig. 15 depicts a different cross section of the same combustion transformation process, as for Fig. 14.

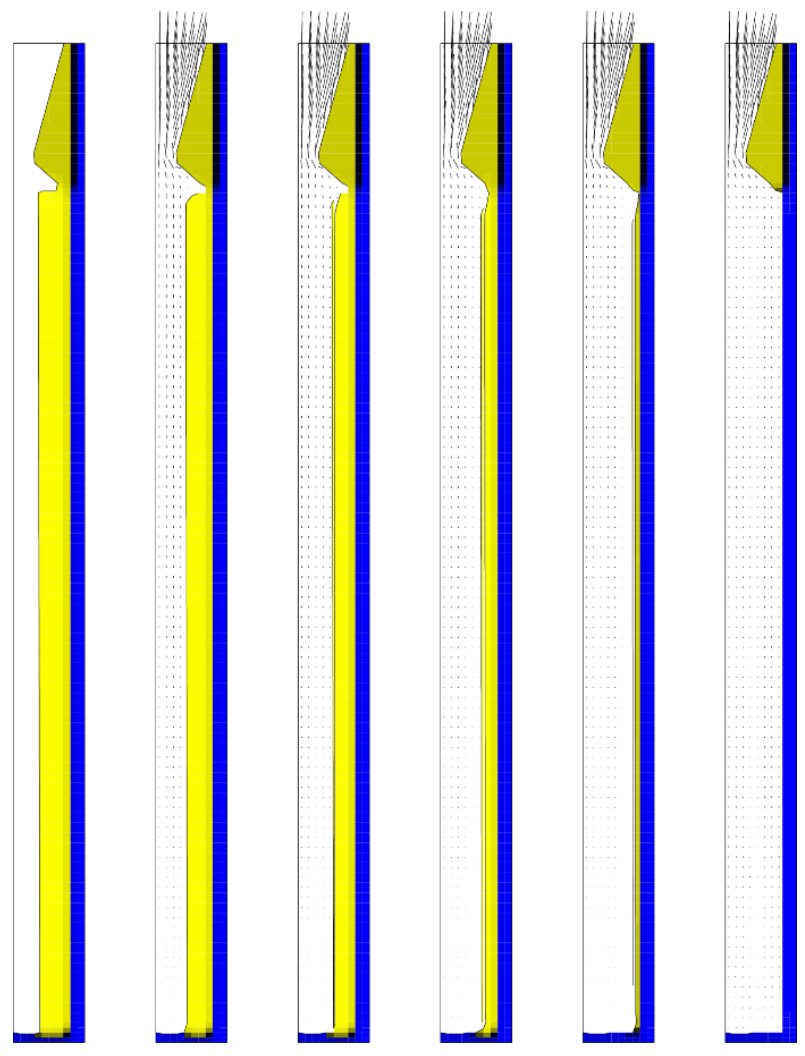

Fig. 15 Propellant combustion evolution at $t=0,1,2,3,4$, and 5 seconds 


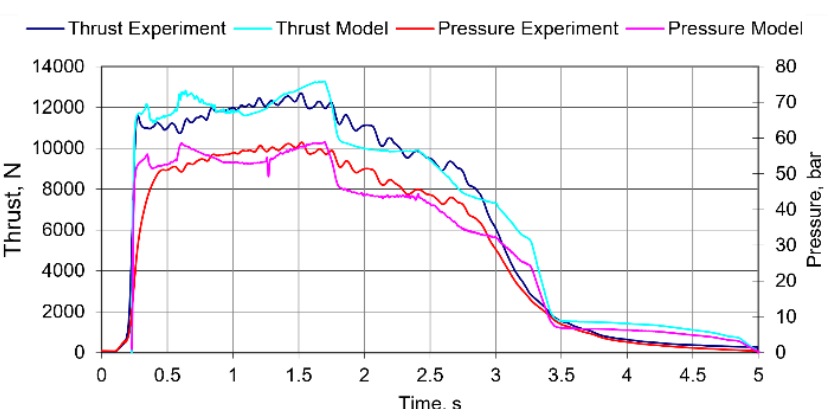

Fig. 16 Thrust and pressure comparison of the rocket motor by the experimental approach correlated with the data of the simulation

\section{Conclusions}

In conclusion, the numerical methods-based simulations are a viable option for the design and development of solid propellant rocket motors, and in some cases are even more advantageous than static testing.

1. This research paper describes the method with regard to numerical simulation of the dynamically changing solid propellant grain domain which is crucial in a solid propellant rocket motor design process.

2. The mesh of the computational domain was cylindrical, structured and based on hexahedral elements, where the entire computational domain consists of 162000 computational cells.

3. Simulation results were obtained using 8 core, workstation grade machine in which the simulation took 714.36 hours to complete.

4. The generated data was post processed and plotted into graphs. In addition, the authors have captured evolution of the combusting solid propellant grain at a specific time interval and depicted them to acquire a better insight of the combustion process itself.

5. Results were represented as 3 different types of graphs. 1 - probe data which monitor pressure, and temperature over time, 2 - mesh dependent data which represents an average fluid temperature, open surface of combusting propellant and volume of the propellant over time. 3 - derived plots which represent the burning rate of the propellant and the thrust produced by the rocket motor.

6. Data from Probe 1, that is positioned near the motor's cap and represents pressure and temperature, has shown readings of 25 bar and of $2242 \mathrm{~K}$ in average respectively. Additionally, the data from Probe 2, positioned at the nozzle exit and representing pressure and temperature, has shown readings of 0.83 bar and $1363 \mathrm{~K}$ in average respectively.

7. The average fluid temperature in the entire domain was $2080 \mathrm{~K}$, the open surface of the propellant grain, excluding sliver phase, was $0.36 \mathrm{~m}^{2}$ in average, the propellant grain volume, excluding sliver phase was regressing steadily in a linear-like manner which has shown a stable combustion behaviour.

8 . Average burn rate of the motor was $0.01 \mathrm{~m} / \mathrm{s}$ and the thrust produced by the motor was $9891 \mathrm{~N}$ in average.

9. The model was capable to accurately capture the entire combustion process including the sliver phase which provided significant additional benefits for the future development and optimization of the rocket motor RM-12K.

In conclusion, it is possible to reproduce static test data using numerical methods based on computer modelling and simulations. This approach provides additional insights that are useful for a better understanding of the processes involved in combustion related problems and the internal ballistics of solid propellant rocket motors. This enables the optimization of the design parameters with minimal cost and maximum benefit.

\section{Acknowledgments}

This research was funded by the Research Council of Lithuania (Science grant No. S-MIP-17-94).

\section{References}

1. Marciniak, B.; Okninski, A.; Bartkowiak, B.; Pakosz, M.; Sobczak, K.; Florczuk, W.; Rarata, G. 2018. Development of the ILR-33 “Amber" sounding rocket for microgravity experimentation, Aerospace Scienceand Technology 73: 19-31.

https://doi.org/10.1016/j.ast.2017.11.034.

2. Altman, D.; Holzman, A. 2007. Overview and history of hybrid rocket propulsion, Progress in Astronautics and Aeronautics 218: 1. https://doi.org/10.2514/4.866876.

3. Ki, W.; Ko, T.; Kim, S.; Yoon, W. 2017. 3D grain burnback analysis using the partial interface tracking method, Aerospace Science and Technology 68: 58-67. https://doi.org/10.1016/j.ast.2017.04.023.

4. Hartfield, R.; Jenkins, R.; Burkhalter, J.; Foster, W. 2003. A review of analytical methods for solid rocket motor grain analysis, 39th AIAA /ASME/SAE/ASEE Joint Propulsion Conference and Exhibit 39: 4506. https://doi.org/10.2514/6.2003-4506.

5. LIANG, G. 2019. Service life prediction of AP/Al/HTPB solid rocket propellant with consideration of softening aging behaviour, Chinese Journal of Aeronautics 32(2): 361-368. https://doi.org/10.1016/j.cja.2018.08.003.

6. Tian, H.; Li, Y.; Li, C.; Sun, X. 2017. Regression rate characteristics of hybrid rocket motor with helical grain, Aerospace Science and Technology 68: 90-103. https://doi.org/10.1016/j.ast.2017.05.006.

7. Pralits J.; Natali D.; Guerrero J.; Bottaro A. 2018. Wolf dynamics - multiphysics simulations, optimization, and data analytics. [accessed 9 Feb. 2019]. Available from Internet: http://www.wolfdynamics.com/ourservices/hpc-cloud-computing.html.

8. Guthrie, J. J. 2015. CFD simulations on a Raspberry Pi cluster. Frankfurt am Main. https://doi.org/10.13140/RG.2.1.1079.2486

9. Lichtl, A.; Jones, S. 2015. GPUs to Mars: Full scale simulation of SpaceX's Mars rocket engine. GTC2015. [accessed 3 Mar. 2019]. Available from Internet: http://on-demand.gputechconf.com/gtc/2015/presentation/S5398-Stephen-Jones-Adam-Lichtl.pdf.

10. Fedaravicius, A.; Survila, A.; Rackauskas, S.; Kilikevicius, S.; Slizys, E.; Patasiene, L. 2017. Air target for a rocket with infrared targeting system, The state Patent Bureau of the Republic of Lithuania. Patent No. 6371.

11. Lyons, J. W.; Long, D.; Chait, R. 2006. Critical technology events in the development of the Stinger and 
Javelin Missile Systems: Project hindsight revisited, National Defence Univ. Washington DC Center for Technology and National Security Policy. [accessed 15 Jan. 2018]. Available from Internet:

https://apps.dtic.mil/dtic/tr/fulltext/u2/a454087.pdf.

12. Lekstutis, A. GUIPEP-Graphical User Interface to PEP.

13. Beckstead, M. W.; Derr, R. L.; Price, C. F. 1970. A model of composite solid-propellant combustion based on multiple flames, AiAA Journal, 8(12): 2200-2207. https://doi.org/10.2514/3.6087.

14. Cummings, R. M.; Mason, W. H.; Morton, S. A.; McDaniel, D. R. 2015. Applied computational aerodynamics. A modern engineering approach. Cambridge University Press 53.

15. Hirt, C. W.; Nichols, B. D. 1981. Volume of fluid (VOF) method for the dynamics of free boundaries, Journal of computational physics 39(1): 201-225. https://doi.org/10.1016/0021-9991(81)90145-5.

16. Hirt, C. W.; Bronisz, B. D. 1991. On the computation of highly viscous flows, Flow Science Technical Note 31 .

17. Van Leer, B. 1997. Towards the ultimate conservative difference scheme, Journal of Computational Physics 135(2): 229-248. https://doi.org/10.1006/jcph.1997.5704.

18. Solid propellant combustion modeling Flow-3d blog [Web log post]. 2017. Retrieved September 10, 2018, from https://www.flow3d.com/solid-propellant-combustion-modeling/

19. Hirt, C. W. 1989. Flow in a Solid-Propellant Rocket Chamber, Flow Science Technical Note \#17.
S. Račkauskas, A. Fedaravičius, A. Survila

\section{NUMERICAL STUDY ON INTERNAL BALLISTICS CHARACTERISTICS OF A SOLID PROPELLANT ROCKET MOTOR}

S u m m a r y

The viability of numerical simulations to design a solid propellant rocket motor, as opposed to static testing, is discussed in this paper. The results demonstrate that an objectively more detailed insight into the processes taking place within the motor in active combustion can be gained by employing computer simulations. The abundance of readily available computing power allows complex simulations to be run on personal desktop computers, thus, saving money by eliminating the need for static testing during the design phase of the motor. The computer simulation results are validated by a comparison with test data gathered during static tests on the motor. In this study, an RM-12K solid propellant rocket motor is designed and developed, which is currently used in real-world air defence training applications, and therefore, sufficient empirical data are available. The numerical method, based on computer simulations using personal computers, is sufficiently accurate to allow for motor design decisions and an adequate substitute for static testing. Even though a single simulation can take up to 715 $\mathrm{h}$ on an 8-core personal machine, it is still an effective solution.

Keywords: rocket motor, solid propellant, internal ballistics, numerical methods. 